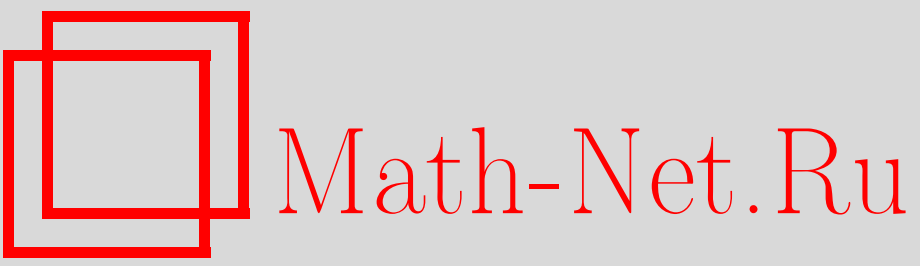

А. В. Топчий, Многомерные непараметрические методы для двухвыборочной задачи сдвига, УМН, 2002, том 57, выпуск 3, 163-164

DOI: https://doi.org/10.4213/rm525

Использование Общероссийского математического портала Math-Net.Ru подразумевает, что вы прочитали и согласны с пользовательским соглашением

http://www.mathnet.ru/rus/agreement

Параметры загрузки:

IP : 54.147 .182 .235

26 апреля 2023 г., $17: 48: 37$ 


\title{
МНОГОМЕРНЫЕ НЕПАРАМЕТРИЧЕСКИЕ МЕТОДЫ ДЛЯ ДВУХВЫБОРОЧНОЙ ЗАДАЧИ СДВИГА
}

\author{
А. В. Топчий
}

1. В работе рассматривается двухвыборочная задача сдвига: есть две независимые $k$-мерные выборки $x_{1}, \ldots, x_{m}$ и $y_{1}, \ldots, y_{n}$ из распределений с функциями распределения $F_{1}(x)=F(x)$ и $F_{2}(x)=F(x-\Delta)$ соответственно, где $F$ - некоторое непрерьвное распределение и $\Delta$ - неизвестный параметр сдвига. Наша задача - оценить неизвестньй параметр сдвига $\Delta$ и проверить нулевую гипотезу $H_{0}: \Delta=0$.

2. Дадим основные определения. Пусть $V\left(\varepsilon_{1}, \ldots, \varepsilon_{k+1}\right)$ - (неориентированньй) объем $k$-мерного симплекса, вершинами которого являются точки с координатами $\varepsilon_{1}, \ldots, \varepsilon_{k+1}$.

ОПредЕлЕниЕ 1 . Оценку $\widehat{\theta}_{m n}$ параметра $\Delta$ определим следующей формулой:

$\widehat{\theta}_{m n}=\arg \min _{\theta \in \mathbb{R}^{k}} U_{m n}(\theta)$, где $U_{m n}(\theta)=\left(C_{n}^{k} C_{m}^{k}(k !)^{2}\right)^{-1} \sum_{(i, j)} V\left(\theta, y_{j_{1}}-x_{i_{1}}, \ldots, y_{j_{k}}-x_{i_{k}}\right)$

и суммирование берется по всевозможным наборам $(i, j)=\left(i_{1}, \ldots, i_{k}, j_{1}, \ldots, j_{k}\right)$ таким, что как среди индексов $i_{1}, \ldots, i_{k}$, так и среди индексов $j_{1}, \ldots, j_{k}$ нет одинаковых.

Оценка $\widehat{\theta}_{m n}$ аффинно инвариантна и в одномерном случае ее значение совпадает с оценкой Ходжеса-Лемана [1]. Она является медианой Оя [2] всевозможных попарных разностей элементов двух выборок.

Сформулируем условия состоятельности и асимптотической нормальности. Для этого введем дополнительные обозначения. Положим $D(\theta)=\mathrm{E} V\left(\theta, y_{1}-x_{1}, \ldots, y_{k}-x_{k}\right)$ и будем предполагать, что $D(\theta)$ определено для всех $\theta \in \mathbb{R}^{k}$. Пусть $\Theta_{0}-$ множество точек, на котором функция $D(\theta)$ достигает свой минимум, т.е. для всех $\theta_{0} \in \Theta_{0}$ и всех $\theta \in \mathbb{R}^{k} D\left(\theta_{0}\right) \leqslant D(\theta)$.

Пусть вектор $d(i, j)=\left(d_{1}(i, j), \ldots, d_{k}(i, j)\right)^{T}$ и скаляр $d_{0}(i, j)$ определяются из следующего разложения:

$V\left(\theta, y_{j_{1}}-x_{i_{1}}, \ldots, y_{j_{k}}-x_{i_{k}}\right)=\frac{1}{k !}\left|\operatorname{det}\left(\begin{array}{ccccc}1 & 1 & \cdots & 1 \\ \theta^{1} & y_{j_{1}}^{1}-x_{i_{1}}^{1} & \cdots & y_{j_{k}}^{1}-x_{i_{k}}^{1} \\ \vdots & \vdots & \ddots & \vdots \\ \theta^{k} & y_{j_{1}}^{k}-x_{i_{1}}^{k} & \cdots & y_{j_{k}}^{k}-x_{i_{k}}^{k}\end{array}\right)\right|=\left|d_{0}(i, j)+d^{T}(i, j) \theta\right|$.

Другими словами, $d_{0}(i, j)=(k !)^{-1} \operatorname{det}\left(y_{j_{1}}-x_{i_{1}}, \ldots, y_{j_{k}}-x_{i_{k}}\right), d_{l}(i, j)=(k !)^{-1} A_{l}$, где $A_{l}-$ алгебраическое дополнение к $\theta^{l}$ в вышеприведенной матрице, $l=1, \ldots, k$. Пусть теперь $(i, j)=$ $(1, \ldots, k, 1, \ldots, k)$ и определим $\Lambda\left(x_{1}\right)=\mathrm{E}\left(d(i, j) \operatorname{sign}\left(d_{0}(i, j)+d^{T}(i, j) \theta_{0}\right) \mid x_{1}\right)$. Положим $\Gamma=$ $\operatorname{cov}\left(\Lambda\left(x_{1}\right), \Lambda\left(x_{1}\right)\right)$.

ТЕОРема 2. Пусть $x_{1}, \ldots, x_{m}, y_{1}, \ldots, y_{n}-$ две независимые выборки из распределений $F(x)$ и $F(x-\Delta)$ соответственно, множество $\Theta_{0}$ состоит из единственной точки $\theta_{0}$ и верно хотя бы одно из следующих двух условий:

(i) $\mathrm{E}\left\|x_{1}\right\|<\infty$ и для некоторьх чисел $\alpha_{1}$, $\alpha_{2}$ вилолнено $0<\alpha_{1} \leqslant m / n \leqslant \alpha_{2}<\infty$;

(ii) $\mathrm{E}\left\|x_{1}\right\|^{r}<\infty$ для некоторого $r>1$.

Тогда $\theta_{0}=\Delta u \widehat{\theta}_{m n} \rightarrow \Delta$ n.н. при $M:=\min \{m, n\} \rightarrow \infty$.

Пусть вместо условий (i) u (ii) выполнено более сильное условие $\mathrm{E}\left\|x_{1}\right\|^{2}<\infty, a$ также дополнительно $\mathrm{P}\left(\operatorname{det}\left(y_{1}-x_{1}-\theta_{0}, \ldots, y_{k}-x_{k}-\theta_{0}\right)=0\right)=0$ и в некоторой окрестности точки $\theta_{0}$ верно разложсение

$$
D(\theta)=D\left(\theta_{0}\right)+\frac{1}{2}\left(\theta-\theta_{0}\right)^{T} A\left(\theta-\theta_{0}\right)+o\left(\left\|\theta-\theta_{0}\right\|^{2}\right),
$$


где $A$ - некоторая положительно определенная матрица. Тогда распределение случайной величинь $\sqrt{\frac{m n}{m+n}}\left(\widehat{\theta}_{m n}-\Delta\right)$ слабо сходится $\kappa k$-мерному нормальному распределению с вектором средних 0 и ковариационной матрицей $\Sigma=k^{2} A^{-1} \Gamma A^{-1}$.

Доказательство теоремы см. в работе [3].

3. Проверка гипотезы об однородности $H_{0}: \Delta=0$.

ОПРЕДЕЛЕНИЕ 3 . Введем тестовую статистику $T_{m n}=\nabla U_{m n}(0)$ (вектор частных производных по $\theta$ в точке $\theta=0)$ и определим $\Psi_{N}=m n(m+n)^{-1} k^{-2} T_{m n}^{T} \Gamma_{N}^{-1} T_{m n}$, где $\Gamma_{N}$ (см. [3]) - некоторая состоятельная оценка матрицы $\Gamma$.

Критерий $T_{m n}$ является многомерным обобщением критерия Уилкоксона, а статистика $\Psi_{N}$ обладает свойством аффинной инвариантности и асимптотически свободна от распределения исходных данных $F$.

ТЕОРема 4 (об асимптотическом распределении статистик $T_{m n}$ и $\Psi_{N}$ при нулевой гипоте-

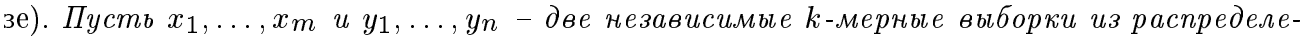
ний с функциями распределения $F_{1}(x)=F(x)$ и $F_{2}(x)=F(x-\Delta)$ соответственно. Пусть также $\mathrm{E}\left\|x_{1}\right\|^{2}<\infty, \mathrm{P}\left(\operatorname{det}\left(y_{1}-x_{1}, \ldots, y_{k}-x_{k}\right)=0\right)=0$ и существует предел $\lambda=\lim _{N \rightarrow \infty} m / N(0<\lambda<1)$, где $N=m+n$. Тогда при нулевой гипотезе $H_{0}: \Delta=0$ предельное распределение статистики $\sqrt{N} T_{m n}-k$-мерное нормальное с нулевьм вектором средних и ковариачионной матрицей $\frac{k^{2}}{\lambda(1-\lambda)} \Gamma$, а предельное распределение статистики $\Psi_{N}$ - чентральное $\chi^{2}$-распределение с $k$ степенями свободы.

ТЕОРема 5 (об асимптотическом распределении статистик $T_{m n}$ и $\Psi_{N}$ при последовательности близких гипотез $H_{N}: \Delta_{N}=N^{-1 / 2} \delta$ ). При некоторьх слабых условиях (сформулированных в работе [3]) асимптотическое распределение случайной величины $N^{1 / 2} T_{m n}$ при альтернативе $H_{N}$ - $k$-мерное нормальное с вектором математических ожиданий $A \delta$ и ковариационной матрицей $\frac{k^{2}}{\lambda(1-\lambda)} \Gamma$. Предельное распределение статисти$\kappa и \Psi_{N}$ при гипотезе $H_{N}$ - нецентральное $\chi^{2}$-распределение с $k$ степенями свободы $и$ параметром нещентральности $\lambda(1-\lambda) k^{-2} \delta^{T} A^{T} \Gamma^{-1} A \delta$.

Как следствие теоремы 5 , мы получаем, что асимптотическая относительная эффективность по Питману критерия $\Psi_{N}$ по отношению к статистике Хотеллинга $T^{2}$

$$
e\left(\Psi_{N}, T^{2}\right)=k^{-2} \delta^{T} A^{T} \Gamma^{-1} A \delta /\left(\delta^{T} \Sigma_{0}^{-1} \delta\right),
$$

где $\Sigma_{0}$ - ковариационная матрица исходного распределения $F$. Для гауссовских распределений (когда критерий $T^{2}$ является оптималшным) эффективность превышает значение 0.955 и возрастает к 1 с увеличением размерности пространства $k$. Для распределений с тяжельми хвостами эффективность $e\left(\Psi_{N}, T^{2}\right)$ может достигать сколь угодно больших значений. В работе [3] приведены значения асимптотических эффективностей $e\left(\Psi_{N}, T^{2}\right)$ для нормального распределения и распределений Стьюдента с различными степенями свободы и размерностями $k$. Те же значения получаются для асимптотических относительных эффективностей (отношений обобщенных дисперсий) оценки сдвига $\widehat{\theta}_{m n}$ по отношению к оценке, которая является разностью выборочных средних двух выборок $n^{-1} \sum y_{j}-m^{-1} \sum x_{i}$ и оптимальна в классе нормальных распределений.

\section{СПИСОК ЛИТЕРАТУРЫ}

[1] J.L. Hodges, E. L. Lehmann // Ann. Math. Statist. 1963. V. 34. P. 598-611. [2] Н. Oja // Statist. Probab. Lett. 1983. V. 1. P. 327-332. [3] А. В. Топчий, Ю. Н. Тюрин, Х. Оя. Многомерные афффинно-инвариантные методы для двухвыборочной задачи сдвига // Рукопись деп. в ВИНИТИ № 2507-В2001, 2001. 